\title{
Inhibition of laser induced rats choroidal neovascularization by intravitreous injection of sEphB4-HSA
}

\author{
Shikun $\mathrm{He}^{1 \# \wedge}$, Sha Ouyang ${ }^{1,2 \#}$, Xiaohua $\mathrm{Li}^{3,4}$, Binyun $\mathrm{Ma}^{5}$ \\ ${ }^{1}$ Department of Pathology and USC Roski Eye Institute, Keck School of Medicine of the University of Southern California, Los Angeles, CA, \\ USA; ${ }^{2}$ Department of Ophthalmology, the Third Xiangya Hospital, Central South University, Changsha, China; ${ }^{3}$ Department of Ophthalmology, \\ Henan Provincial People's Hospital, Zhengzhou, China; ${ }^{4}$ Department of Ophthalmology, Henan Eye Hospital, Zhengzhou, China; ${ }^{5}$ Department of \\ Medicine, Keck School of Medicine of the University of Southern California, Los Angeles, CA, USA \\ Contributions: (I) Conception and design: S He, B Ma; (II) Administrative support: None; (III) Provision of study materials or patients: None; (IV) \\ Collection and assembly of data: S He, S Ouyang, B Ma; (V) Data analysis and interpretation: All authors; (VI) Manuscript writing: All authors; (VII) \\ Final approval of manuscript: All authors. \\ \#These authors contributed equally to this work. \\ Correspondence to: Shikun He, MD. USC Roski Eye Institute, Keck School of Medicine of the University of Southern California, Los Angeles, 1450 \\ San Pablo St, \#4400, Los Angeles, CA 90033, USA. Email: shikunhe@usc.edu; Binyun Ma. Department of Medicine, Keck School of Medicine of the \\ University of Southern California, Los Angeles, CA 90033, USA. Email: binyunma@usc.edu.
}

Background: Choroidal neovascularization $(\mathrm{CNV})$ is a leading cause of central vision loss complicated with age-related macular degeneration. Although intravitreal anti-VEGF therapy is widely used in wet age-related macular degeneration, optimal treatment regimens for the disease are still under investigation. EphrinB2 and EphB4 regulate angiogenesis, and interruption of EphB4/ephrinB2 has been demonstrated to inhibit angiogenesis. In the current study, we studied the effects of soluble EphB4 (sEphB4) on laser induced $\mathrm{CNV}$ in a rat model by intravitreous injection and the underlying mechanism.

Methods: Male rats (Brown-Norway) were used in the study. CNV was induced by laser and the sEphB4 was injected intravitreous after laser at days 3 and 7. The CNV lesions were evaluated by three methods: fluorescein angiography (FA) in vivo, CNV volume by confocal analysis of choroidal flat-mounts and H\&E staining. The expression of fibronectin (FN), VEGFR-2, phospho-VEGFR-2 (pVEGFR-2), the double labeling of EphB4 with FN was analyzed by immunofluorescence. The interaction of FN with EphB4 and the effects of intraocular injection of sEphB4 on the inhibition of pVEGFR-2 were determined by western blot.

Results: The FA leakage and CNV volume were significantly inhibited by the injection of the sEphB4. Further, histology analysis showed that CNV lesion was significantly smaller in the rats with sEphB4 injection than rats with placebo application. The expressions of pVEGFR-2 and FN in the CNV lesions were reduced compared with controls.

Conclusions: Our study suggests that the inhibition of CNV by sEphB4 may be through suppression of VEGFR-2 phosphorylation and the expression of FN. sEphB4 may be a new potential therapeutic strategy of CNV.

Keywords: Choroidal neovascularization (CNV); soluble EpHB4 (sEphB4); VEGF-R2; fibronectin (FN)

Submitted May 08, 2020. Accepted for publication Sep 30, 2020.

doi: $10.21037 / \mathrm{atm}-20-3810$

View this article at: http://dx.doi.org/10.21037/atm-20-3810

^ ORCID: 0000-0002-0797-9149. 


\section{Introduction}

Choroidal neovascularization (CNV) is the formation of new blood vessels from the choroid capillaries into the subretinal space (1). It is a common complication of agerelated macular degeneration, and leads to irreversible central vision loss $(1,2)$. The pathogenesis of $\mathrm{CNV}$ is associated with the upregulation of cytokines (IL-1 $\beta$, IL-6, IL-10, IL-18 and TNF- $\alpha$ ) (3) and growth factors (PDGF, VEGF, bFGF, IGF-1, CTGF and TGF- $\beta$ ) (4) and overproduction of extracellular matrix components. The critical growth factor in the induction of CNV is VEGF (5). High expression of VEGF in CNV has been demonstrated in vivo and in vitro studies (6), and therefore, the role of VEGF in the induction of $\mathrm{CNV}$ is well recognized.

Anti-VEGF agents are widely used in the treatment of $\mathrm{CNV}$ due to the critical role of VEGF in the pathogenesis of angiogenesis (7). In spite of the varying success of anti-VEGF treatments, there are a number of caveats to consider. Up to $32 \%$ of patients are non-responsive to anti-VEGF treatment despite monthly injections (8). In such cases; progression of the disease may be featured with subretinal hemorrhage and persistent macula fluid, all which can contribute to central vision loss. Even for patients that respond to VEGF treatment, there are safety concerns regarding the possibility of increased arteriothrombotic events (9). For patients with severe proliferative diabetic retinopathy, application of bevacizumab has been complicated with tractional retinal detachment (10). Additionally, higher doses of anti-VEGF agents have been associated with uveitis (11).

The adverse side-effects of anti-VEGF treatments have led the search for alternative therapeutic approaches. Targeting Ephrin receptors-EphB4 has been recognized as an effective method in the inhibition of tumor and eye neovascularization (12). EphB4 is a receptor tyrosine kinase, and its cognate transmembrane ligand EphrinB2 regulates angiogenesis (13). In embryos, Eph/ephrin plays a role in cellular positioning and pattern formation (14). Notably, EphrinB2 is the only ligand for EphB4, but the Ephrin B2 can activate several EphB receptors (15). The binding of Eph receptor and its ligand results in receptor clustering and activation of receptor signaling pathways in both directions (16). EphrinB2 and EphB4 expression in arterial and venous endothelium respectively regulates vascular remodeling (17) and their disruption inhibits the formation of new blood vessels (18).

EphB4 and its ligand EphrinB2 are expressed in the choroidal vessels of adult rats, and regulate neovascularization (19). Furthermore, human retinal endothelial cells express ephrinB2 and EphB4; EphrinB2 has been found to be expressed in endothelial cells in human specimen with diabetic retinopathy and premature retinopathy (20). Our previous studies have shown that soluble EphB4, the extracellular domain of EphB4, is an effective antagonist of EphB4/EphrinB2 signaling due to its high affinity binding to EphrinB2 (21). Rat CNV was inhibited by injection of sEphB4 (19), and sEphB4 has been shown to inhibit PDGF-induced proliferation of retinal pigment epithelium in vitro (21). Those results suggest that $\mathrm{sEphB} 4$ is a promising therapeutic reagent for the treatment of $\mathrm{CNV}$; however, the mechanism of the inhibition of $\mathrm{CNV}$ formation by sEphB4 still under investigation, and also efficacy could be improved further by modifications that increase the half-life of the reagent. Full length albumin coding sequence in frame at the C-terminal of sEphB4 results in fusion protein of $129 \mathrm{kD}$ that has a long halflife (Figure 1A) and thus improved efficacy in mouse tumor models (22). This therapeutic is now in clinical trials for metastatic cancer (clinical trial numbers: NCT02717156; NCT02767921; NCT03049618; NCT03146971; NCT02495896; NCT01642342; NCT03049618). Therefore, in this study, we sought to determine the mechanism of the inhibition of laser induced rat $\mathrm{CNV}$ by intravitreous injection of the sEphB4. The following three primary outcome measures were analyzed: Fluorescein angiograms, $\mathrm{CNV}$ volume and histologic examinations. In addition, the expressions of FN and Phospho-VEGFR2 were evaluated as the secondary outcome measures after sEphB4 injection. Laser induced rat $\mathrm{CNV}$ is one of well-recognized and typic method for the study of the pathogenesis of $\mathrm{CNV}$, the animals recapitulate several key features of experimental CNV. The critical point is that this kind of model cannot be produced in human. Because the possible effect of hormone on the formation of $\mathrm{CNV}$, the male rats were used in the study (23). Due to EphrinB2 and $\mathrm{EphB} 4$ are more extensive involved in the regulation of neovascularization than VEGF (24) and the successful implementation of this study not only provide a further theoretical basis for soluble EphB4 treatment of human $\mathrm{CNV}$, but also have general guiding significance for the treatment of other neovascular eye diseases.

We present the following article in accordance with the ARRIVE reporting checklist (available at http://dx.doi. org/10.21037/atm-20-3810). 


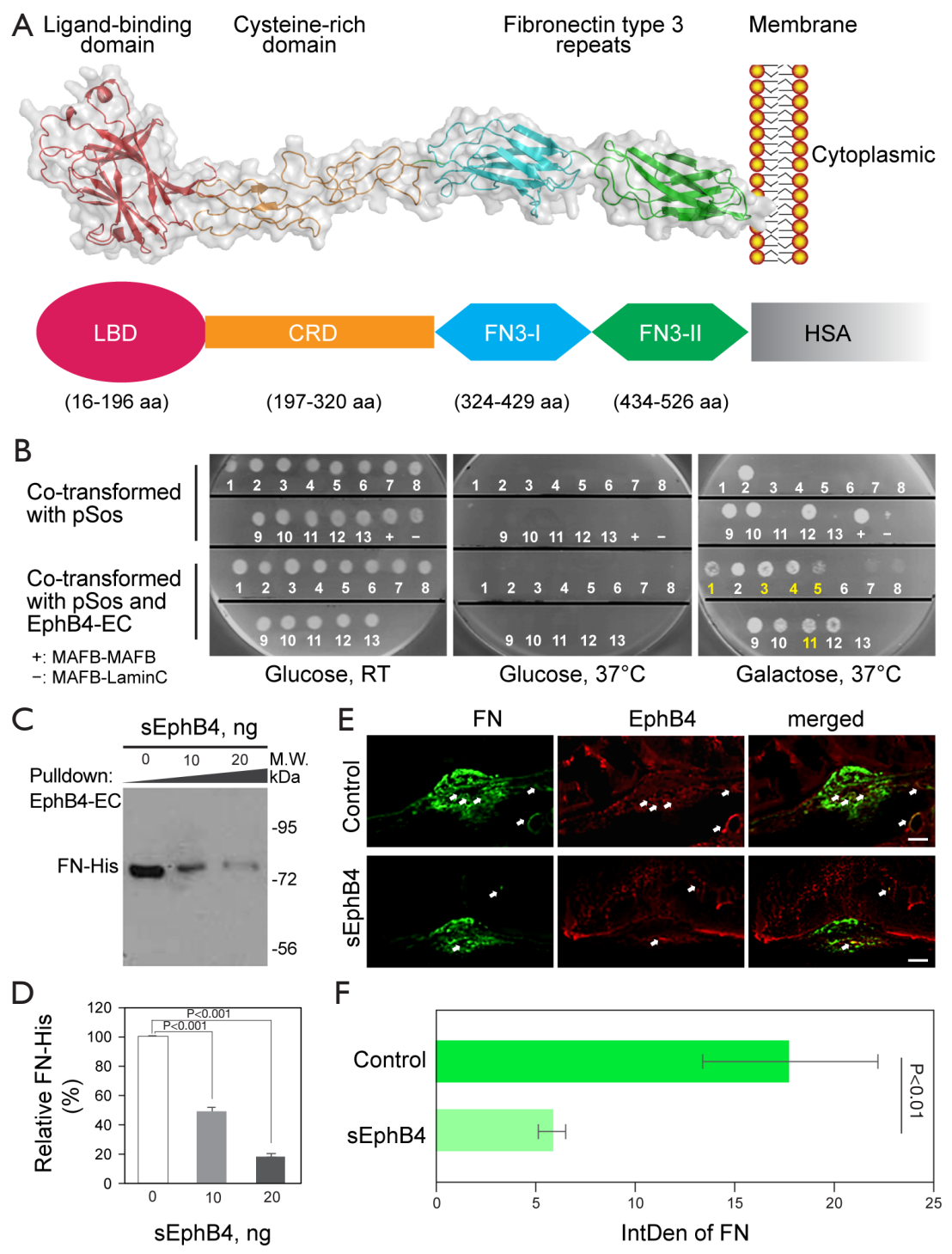

Figure 1 Human EphB4 extracellular domain and the interaction of EphB4 and FN (fibronectin). (A) Diagram of human sEphB4 extracellular domain (EphB4-EC) and soluble sEphB4-HSA (sEphB4-HSA). Top, sEphB4 (human EphB4 extracellular domain) with a ligand binding globular domain (LBDG), a cysteine rich domain (CRD), and two fibronectin type FN 3-like domains (FFN3-I and FN3II). Bottom, sEphB4-HSA that has human serum albumin (HSA) fused to the C-terminus of human EphB4-ECsEphB4. (B) Interaction of EphB4 with FN. Interaction partners of EphB4 extracellular domain (B4-EC) were identified using Cyto Trap yeast two-hybrid system. The positive clones were co-transformed with $\mathrm{B} 4-\mathrm{EC}$ to validate their interaction. The validated clones can grow at $37^{\circ} \mathrm{C}$ on galactose containing (SG) plate only in the presence of B4-EC co-expression. Among the five validated positive clones, \#1, 4, 5, and 11 are FN clone variants, whereas \#3 is a different gene. (C,D) Recombinant B4-EC was biotinylated and immobilized on streptavidin beads, followed by incubation with recombinant His-tagged $\mathrm{FN}$ (FN-His) at presence of 0 , 10, and $20 \mathrm{ng} \mathrm{sEphB} 4$. After wash with PBS, the bound FN-His was detected with anti-His tag antibody on Western, and the relative density of FN-His was shown in (D). The double labeling of FN and EphB4 in the laser induced $\mathrm{CNV}$ (days 14, E), green indicates the positive staining of FN and red represents EphB4 immunoreactivity. Yellow is the colocalization of FN and EphB4 (arrows). The intensity of FN staining was significantly reduced in the CNV (choroidal neovascularization) lesion injected with sEphB4-HSA compared with PBS (phosphate-buffered saline) injection (F). Scale bar: $50 \mu \mathrm{m}$. 


\section{Methods}

\section{Animal and reagent}

Adult male pigmented rats (Brown-Norway; Charles River Laboratories, Wilmington, MA) were used in the study, and all procedures were conducted in accordance with the ARVO Statement for the Use of Animals in Ophthalmic and Vision Research and approved by the University of Southern California Animal Use Committee under a project. Extracellular domain of human EphB4 followed by human serum albumin (HSA) coding cDNA was expressed in CHO (Chinese hamster ovary) cells in chemically defined medium. Protein was purified using Blue Agarose beads to homogeneity, host cell protein levels less than $80 \mathrm{ppm}$, endotoxin levels $<0.5 \mathrm{EU} / \mathrm{mg}$. Protein stability was documented at $4{ }^{\circ} \mathrm{C}$ and $-20{ }^{\circ} \mathrm{C}$ up to 3 years.

\section{EphB4 binding with FN}

CytoTrap yeast two-hybrid system: the CytoTrap system uses the yeast $S$. cerevisiae temperature-sensitive mutant strain $\operatorname{cdc} 25 \mathrm{H}$, which contains a point mutation in the CDC25 gene, the yeast homologue of the human Sos (bSos) gene. The $\operatorname{cdc} 25 \mathrm{H}$ strain can only grow at the permissive temperature $\left(25^{\circ} \mathrm{C}\right)$ but not $37^{\circ} \mathrm{C}$. Expression of hSos and its subsequent localization to the plasma membrane allows the cdc $25 \mathrm{H}$ yeast strain to grow at $37^{\circ} \mathrm{C}$. In CytoTrap system, the localization of hSos to the plasma membrane occurs through the interaction of two-hybrid proteins. DNA encoding the protein of interest (bait protein) is cloned into the pSos vector, generating a fusion protein of hSos and the bait protein. DNA encoding another protein of interest (target protein) or an expression library is cloned into the $\mathrm{pMyr}$ vector MCS and expressed as a fusion protein with a myristylation sequence that anchors the fusion protein to the plasma membrane. If the bait and target proteins physically interact, the hSos protein is recruited to the membrane, thereby activating the Ras-signaling pathway and allowing the cdc $25 \mathrm{H}$ yeast strain to grow at $37^{\circ} \mathrm{C}$. The GAL1 promoter drives the expression of the Myr-target fusion protein thus galactose is needed for its expression and the subsequent bait-target interaction and cell survival at $37^{\circ} \mathrm{C}$. Over 20,000 clones were screened and 7 clones were validated. In vitro interaction analysis of $\mathrm{FN}$ and $\mathrm{EphB} 4$ : $1 \mu \mathrm{g}$ biotinylated recombinant EphB4 extracellular domain (B4-EC) was incubated with $10 \mu \mathrm{L}$ Streptavidin beads for $30 \mathrm{~min}$ at $4{ }^{\circ} \mathrm{C}$ in $20 \mathrm{mM}$ Tris buffer containing $150 \mathrm{mM}$ $\mathrm{NaCl}$ and $0.5 \%$ BSA. After 3 washes with PBS, beads were incubated with $0.45 \mu \mathrm{g}$ His-tagged recombinant FN (FNHis) for $45 \mathrm{~min}$, followed by 2 washes with PBS. The bound proteins were eluted with Laemmli sample loading buffer for western blot analysis. Anti-His tag antibody (Roche) was used for FN-His detection.

\section{Laser induced Rat CNV}

The rat CNV model was induced by laser and used to evaluate the effects of the sEphB4 on choroidal angiogenesis in vivo (19). Thirty adult male pigmented rats (Brown-Norway, aged 6-8 weeks with average weight 170 gram) were obtained and acclimatized for at least $48 \mathrm{~h}$. Vendor health reports indicated that the rats were free of known viral, bacterial and parasitic infection. The cages for housing rats were obtained commercially and two rats were randomly housed per cage. The cages were ranked in ascending order, i.e., cage 1, cage 2 till cage 15, etc. consecutively. The rats were kept in a living condition with an inverse 12 hours day-night cycle in a temperature $\left(22 \pm 1{ }^{\circ} \mathrm{C}\right)$ and humidity $(55 \% \pm 5 \%)$ controlled room. All rats were allowed free access to water and a maintenance diet containing $0.75 \%$ calcium (EURodent Diet 22\%; PMI Nutrition Inter national, LLC, Brentwood, MO, USA). The laboratory rats contact bedding was all commercially obtained for hosting the animals. The rats were randomly divided into 5 groups (6 rats/group) including PBS, sEphB4 $(0.2,0.6,2,6 \mu \mathrm{g})$ injection, to minimize the effects of subjective bias, all of investigators and the surgical performer were blind for the grouping of the rats, therefore, the laser and injection procedure were done in ascending order from 1-15 cages. For each injection, the experimental unit was an individual eye. the surgical procedure was performed with sterile technique in a surgery room for laboratory animal. The rats were anesthetized for all procedures with an intramuscular injection of $0.2 \mathrm{~mL}$ of a 50:50 mixture of ketamine hydrochloride $(20 \mathrm{mg} / \mathrm{mL})$ and xylazine hydrochloride $(100 \mathrm{mg} / \mathrm{mL}$; both from Phoenix Pharmaceutical Inc., St. Joseph, MO). The pupils were dilated with $5 \%$ phenylephrine hydrochloride and $0.8 \%$ tropicamide, and four photocoagulation lesions were delivered with a diode red laser $(140 \mathrm{~mW}, 0.05 \mathrm{~s}, 75 \mu \mathrm{m}$ IRIDEX, Mountain View, CA) between the retinal vessels in a peripapillary distribution in each fundus. Production of subretinal bubble at the time of laser treatment confirmed the rupture of Bruch's membrane. Any lesions which did not produce bubble at the time of laser (suggesting no rupture of Bruch's membrane) or lesions with obvious 
bleeding were excluded in the study. The sEphB4 $(10 \mu \mathrm{L}$, containing $0,0.2,0.6,2$ or $6 \mu \mathrm{g}$ soluble protein, Vasgene Therapeutics, Inc., CA), or vehicle (PBS) was injected with a 30-gauge needle (Hamilton, Reno, NV) introduced into the vitreous $200 \mu \mathrm{m}$ posterior to the limbus, 3 and 7 days after laser photocoagulation. Following the surgical procedure, antibiotic ointment was applied to the eyes of each rat and the rats were maintaining in a warm blanket until waking up. The selection of the doses of the injection of sEphB4 was from our previous publication and preliminary experiment (19). Choice of specific anaesthetic reagents used in the experiment mentioned before and route of administration was our regular approach and was approved by University of Southern California Animal Use Committee.

Intravitreal injection is an extensive used and well recognized method for the study the inhibition of $\mathrm{CNV}$ in experimental animal, it is also a regular method for antiVEGF therapy in the treatment of human CNV.

The animals' health status was monitored throughout the experiments by a health surveillance program according to The Guide for the Care and Use of Laboratory Animals (USA, 2011), there was not behavioral abnormal of the rats in the entirely period of the experiment.

\section{Fluorescein angiography (FA)}

FA which indicated the leakage of new vessels was performed on day 14, as described (19). The intensity of staining in late-phase FA (180-300 seconds after fluorescein injection) was scored by two investigators in a blinded (the ID of the FA images for the treatment applied to individual animal was blocked before the images were scored) manner as following 0 , no staining; 1 , slight leakage; 2 , moderate leakage; and 3, heavy leakage using standardized photographs for each stage (19). The mean score was obtained from the individual FA image in each group of sEphB4 injection and compared with PBS injection statistically.

\section{Histologic analysis of CNV lesions}

On days 4 and 14 , after the rats were sacrificed with an overdose of pentobarbital sodium, the eyes were enucleated and snap frozen in optimal cutting temperature solution (Ames/Miles, Elkhart, IN). Each lesion was cryostat sectioned at $6 \mu \mathrm{m}$ intervals and the sections were placed on glass slides coated with poly-L-lysine (Sigma-
Aldrich). The sections were fixed in acetone for 5 minutes and hematoxylin and eosin (H\&E) staining was used to evaluate $\mathrm{CNV}$ formation. The new vessel and fibrovascular membrane through Bruch's membrane and the monolayer of RPE into the subretinal space was an indicator of CNV formation after laser application.

The CNV thickness and area from multi-retinal sections (stained with $\mathrm{H} \& \mathrm{E}$ ) and rats were analyzed using the software of Image J (NIH, version 1.52). The maximum thicknesses of $\mathrm{CNV}$ and outlined $\mathrm{CNV}$ area were digitized from each $\mathrm{CNV}$ lesion. the morphometric data of $\mathrm{CNV}$ lesions (thickness and area) from each group were averaged to provide a mean value and standard deviation (SD) per group (PBS and sEphB4 injection), respectively. The sEphB4 $(2.0 \mu \mathrm{g})$ was selected for these studies. The data of each group were then calculated using one-way ANOVA analysis followed by Dunnett post hoc test.

\section{Quantitative assessment of CNV lesion size in flat-mount retina}

Rat eyecups were fixed in 4\% paraformaldehyde and then permeabilized in $1 \%$ Triton $\mathrm{X}-100$ for 2 hours. After removal of the anterior segment and neural retina, fluorescein-labeled isolectin B4 (specific endothelial cell marker, 1:100; Vector Laboratories) was added to the eyecup and incubated at $4{ }^{\circ} \mathrm{C}$ overnight. The sections were cover-slipped (Vectashield medium; Vector Laboratories), and the lectin labeling was examined with the $20 \times$ objective of a laser scanning confocal microscope (LSM; model 510; Carl Zeiss Meditec, Dublin, CA). Fluorescence volume measurements were made by creating image stacks of optical slices within lesions. The image stacks were generated in the Z-plane, with the confocal microscope set to excite at $488 \mathrm{~nm}$ and to detect at 505 to $530 \mathrm{~nm}$. Images were further processed using the microscope's system software (LSM; Carl Zeiss Meditec, Inc.) by closely circumscribing and digitally extracting the fluorescent lesion areas throughout the entire image stack. The extracted lesion was processed through the topography software to generate a digital topographic image representation of the lesion and an image volume (square micrometers $\pm \mathrm{SD}$ ) that reflects the CNV fluorescence volume (5).

\section{Immunofluorescence}

The retinal sections were fixed with $4 \%$ formaldehyde in phosphate buffered saline (PBS) for $10 \mathrm{~min}$ at room 
temperature (RT), washed with PBS, and then permeabilized with $0.1 \%$ Tween-PBS for 10 minutes. After a 30 -min blocking with $10 \%$ normal goat serum, the sections were incubated with anti-fibronectin (1:100, Abcam, Cambridge, MA), VEGFR-2 (1:100, Abcam, Cambridge, MA) and pVEGF-R2 (1: 50, Abcam, Cambridge, MA), EphB4 (1:25, SC-130081, Santa Cruz Biotechnology, Inc. Dallas, Texas) overnight at $4{ }^{\circ} \mathrm{C}$ and then fluorescent conjugated secondary antibody (1:200, vector, Burlingame, CA) was applied to the sections for $30 \mathrm{~min}$ at RT. The sections were mounted with propidium iodide mounting medium. For double staining, the sections were incubated with the anti-fibronectin (1:15, Santa Cruz, sc18825, Santa Cruz Biotechnology, Inc. Dallas, Texas) for $1 \mathrm{~h}$ at RT, secondary anti-Mouse antibody (1:150, vector, Burlingame, CA) $30 \mathrm{~min}$ at RT. After washing the sections with PBS, the anti-EphB4 (1:25, Santa Cruz Biotechnology, Inc. Dallas, Texas) was applied to the sections and incubated overnight at $4{ }^{\circ} \mathrm{C}$, followed by secondary anti-Rabbit antibody (1:150, vector, Burlingame, CA) for $30 \mathrm{~min}$ at RT. The double labeled sections were mounted with the mounting medium (without color, Vector Laboratories, Burlingame, CA) and examined with a Keyence fluorescence microscope. The staining intensity of fibronectin in the lesion of laser induced $\mathrm{CNV}$ from multi-retinal sections and rats were analyzed using ImageJ (National Institutes of Health https://imagej.nih.gov/ij/)

\section{Western blot analysis}

The pVEGF-R2 and VEGF-R2 levels in rat eyes treated with/without the sEphB4 were determined with western blot. The rat eye cup protein was prepared with RIPA buffer on ice. Protein concentration was determined by BCA protein assay (Thermo Scientific). Ten $\mu \mathrm{g}$ of the lysate was run on 4-20\% Tris-glycine gradient gel (Bio-Rad, Hercules, CA, USA) at $150 \mathrm{~V}$. The protein was then electrotransferred onto nitrocellulose membrane (Bio-Rad). The membrane was blocked with $5 \%$ BSA in Tris-buffered saline for $1 \mathrm{~h}$, and then incubated with the following primary antibodies at $4{ }^{\circ} \mathrm{C}$ overnight: pVEGF Receptor 2 (Tyr1059) (D5A6) Rabbit mAb (1:1,000, \#3817, Cell Signaling, Danvers, MA), VEGF Receptor 2 (D5B1) Rabbit mAb (1:1,000, \#9698, Cell Signaling Danvers, MA), anti EphB4S antibody H200 (Santa Cruz, SC-5536), and beta-Actin mouse mAb (Sigma) $(1: 2,000)$. Membranes were then incubated with fluorescent secondary antibodies $(1: 10,000)$ for $1 \mathrm{~h}$ and signal was detected using Odyssey System (LI-COR, Lincoln, NE, USA). Signal intensity was quantified with ImageJ (NIH).
VEGF-R2 and pVEGF-R2 level was normalized to betaactin. All these experiments were repeated three times and similar results were obtained. All error bars represent SD.

\section{Statistics analysis}

For the analysis of $\mathrm{FN}$ and $\mathrm{CNV}$ volume, the experimental unit was an individual $\mathrm{CNV}$ lesion in the rat retina and for the evaluation of expression of phosph-VEGFR2 and $\mathrm{H} \& \mathrm{E}$ staining the experimental unit was eyes. All results are expressed as means \pm SD. One-way ANOVA analysis followed by Dunnett post hoc test was performed for the statistics analysis; $\mathrm{P}<0.05$ was accepted as significant.

\section{Results}

\section{EphB4 interaction with FN}

The diagram of the structure of the soluble EphB4 and EphB4-HSA was shown in Figure 1A. Prior pharmacokinetic studies of intravitreal sEphB4 in rabbit showed halflife longer than Lucentis and Avastin (25). We wondered if EphB4 localizes to vessel wall in CNV model. We hypothesized that EphB4 may bind to previously unrecognized proteins which located in the retina resulting in long retention time in the eye. We thus performed yeast two hybrid studies as an unbiased approach to identify partner proteins (Figure 1B). Extracellular EphB4 protein was used as the bait against embryonically expressed gene cDNA library. Most commonly partnering protein was the c-terminal region of FN. This interaction was further confirmed by pulldown experiments (as showed in Figure 1C,D). Fibronectin localizes to the exterior of the newly forming vessel endothelium and pericytes $(26,27)$. Our in vivo experiment showed that the expression of $\mathrm{FN}$ in rats $\mathrm{CNV}$ was significantly reduced after the sEphB4 intravitreous injection (Figure $1 E, F ; \mathrm{P}<0.01$ ), suggesting that the binding of $\mathrm{FN}$ to EphB4 was inhibited by sEphB4.

\section{Suppression of rats CNV by intravitreal injection of $s E p h B 4$}

All rats were free from microbiological infection before and after the surgical procedures, there was no loss of the weight and behavior abnormal of the rats included in the entire of experiment status, To study the effects of intravitreal injection of the $\mathrm{sEphB} 4$ on the development of laser-induced CNV in vivo, the CNV lesion was 

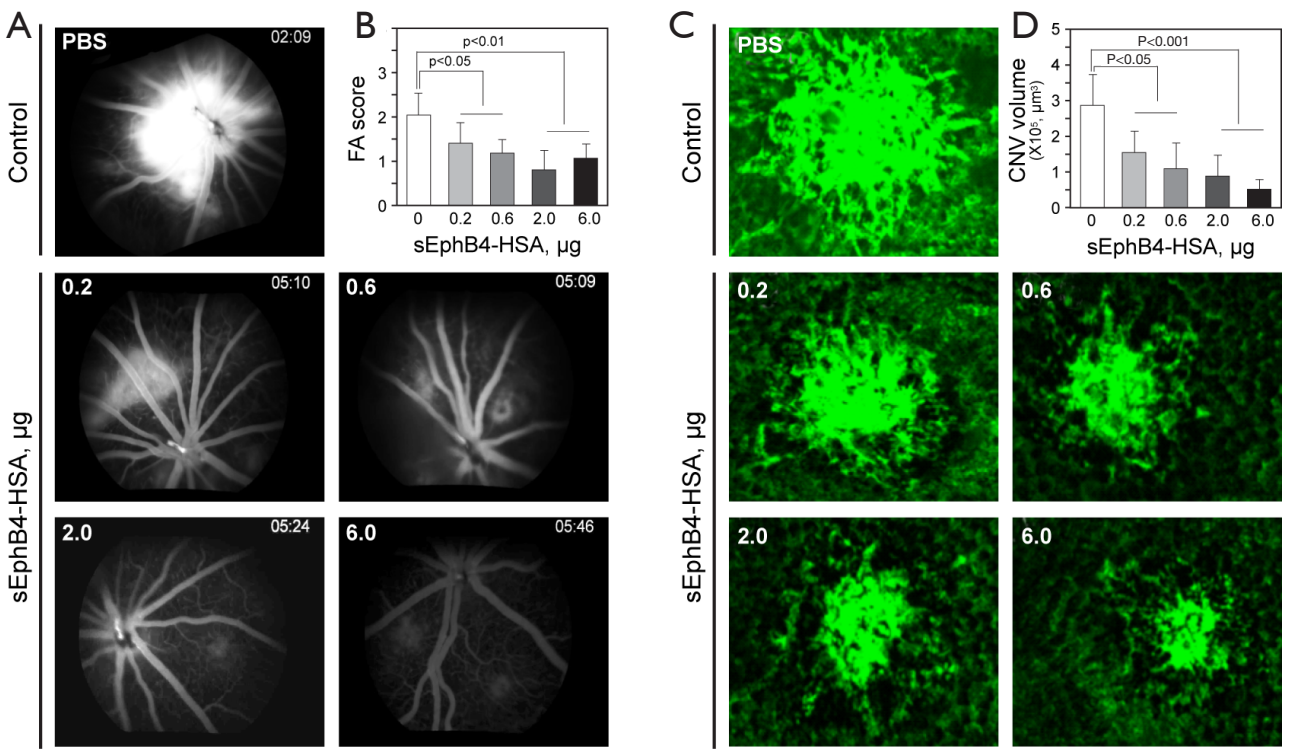

Figure 2 Effects of the sEphB4 injection on fluorescein leakage and CNV (choroidal neovascularization) lesion in rat. Fluorescein angiogram (FA) was performed 14 days after laser photocoagulation and intravitreal injection of the sEphB4. (A) Representative FA images in laser-induced rat (B) FA score: sEphB4 injection resulted in a dose dependent inhibition of fluorescein leakage CNV (20 FA images were pooled in each group). (C) Representative CNV lesions from PBS or different concentrations of sEphB4-HSA injection measured in FITClabeled isolectin-B4 stained flat retinal mounts on day 14. (D) After being stained with isolectin B4, retinal flatmounts were visualized with a laser scanning confocal microscope $\mathrm{Z}$ stack images of $\mathrm{CNV}$ lesion were taken. The image stacks were rendered in $3 \mathrm{D}$ using velocity imaging software (Improvision Inc., Waltham, USA) and processed to digitally extract the fluorescent lesion volume. CNV volume was measured in micrometers cubed (14 images/each group), A dose dependent significant reduction of CNV volume was seen in EphB4-HSA treated rats compared with PBS (phosphate-buffered saline) injection.

evaluated by three methods: FA in vivo, CNV volume by confocal analysis of choroidal flatmounts and histology. At day 14 post-laser, the leakage was assessed by FA, the maximal inhibition of FA leakage could be seen at $2 \mu \mathrm{g}$ the sEphB4 injection compared with control (Figure $2 A, B, \mathrm{P}<0.01$ ). Figure $2 C$ demonstrates a representative $C N V$ lesion from $\mathrm{PBS}$ and various concentrations of the $\mathrm{sEphB} 4$ injection. $\mathrm{CNV}$ volume was also significantly reduced; Figure $2 D$ reveals a dosedependent reduction of $\mathrm{CNV}$ volume in the $\mathrm{sEphB} 4$ treated rats compared to rats with $\mathrm{PBS}$ injections, the strongest inhibition of $\mathrm{CNV}$ volume was seen with the application of 2.0 and $6.0 \mu \mathrm{g} \mathrm{sEphB4}$ (Figure $2 D, \mathrm{P}<0.01$ ).

Figure $3 A$ indicated the schedule and the treatment applied to the rats, while Figure $3 B$ was a diagram of $\mathrm{CNV}$ formation. By analyzing the $\mathrm{H} \& \mathrm{E}$-stained $\mathrm{CNV}$ sections (Figure 3C) using the software Image J, We found that the $\mathrm{CNV}$ area and thickness were significantly reduced in the rats injected with sEphB4 $(2 \mu \mathrm{g})$ compared with PBS application at days $14(\mathrm{P}<0.05,0.01$, respectively)
(Figure 3D,E), however, there was no significant difference in term of the thickness of CNV between the two groups at the days 4 (Figure 3D).

\section{sEphB4 inhibits VEGF-R2 phosphorylation in CNV lesions}

Previous studies have shown that EphrinB2, the target of sEphB4 is induced in newly forming vessels, it binds VEGFR2 and VEGFR3 and is required for VEGFR signaling $(28,29)$. We thus conducted immunostaining of CNV lesion sections for VEGFR2 in the sEphB4 treated and control eyes (Figure 4A). There was no statistical difference in the expression of total VEGFR2 in the sEphB4 treated group and control. However, the immunoreactivity of pVEGFR2 in the sEphB4 injected rats was markedly lower compared to lesions in control rats (Figure 4B). Similar to the immunostaining, Western blot analysis showed that pVEGFR2 was significantly reduced in the retina injected with sEphB4 $(2.0 \mu \mathrm{g})$ compared to vehicle treated controls (Figure $4 C, D, \mathrm{P}<0.05$ ). 


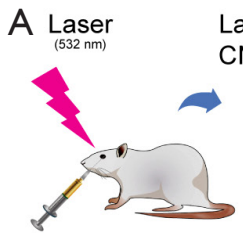

Treatment

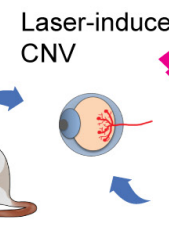

sE
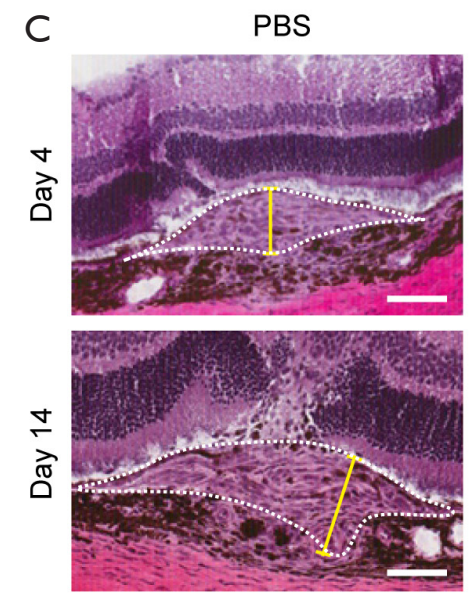

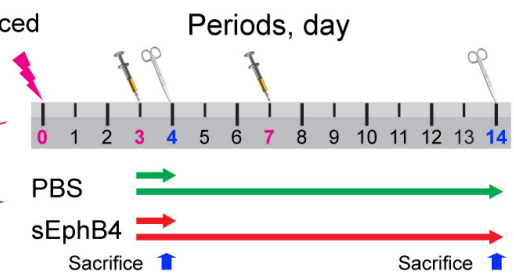

sEphB4-HSA
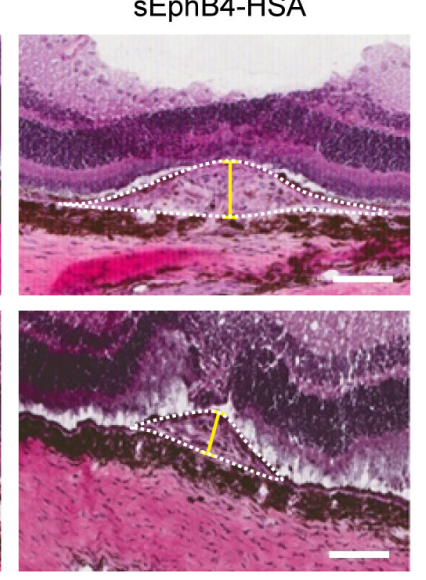

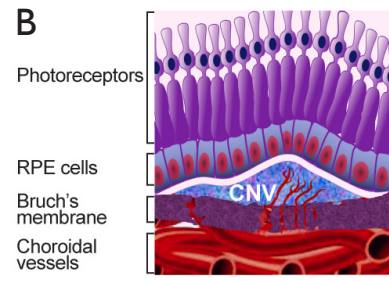

D
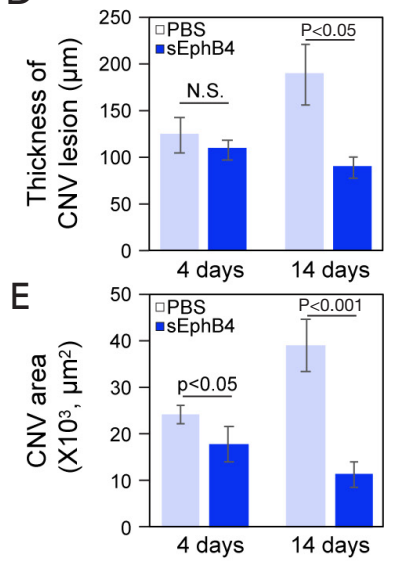

Figure 3 Effects of sEphB4-HSA injections on rat CNV (choroidal neovascularization) formation induced by laser. (A) Schematic diagram of the timeline of the laser application in rats, treatments, and sacrifice. (B) Morphology scheme of laser-induced CNV in rat. (C) A representative light microscopic images of $\mathrm{CNV}$ lesion were obtained at days 4 or 14 after laser application. Hematoxylin \& eosin stain (H\&E) (C), yellow line indicates the maximum thicknesses of CNV and CNV areas are outlined with white dot line. Scale bar: $100 \mu \mathrm{m}$. statistical analysis of thickness of rat CNV lesion (D) and CNV area in (E).

\section{Discussion}

EphB4 has been of interest for the treatment of ocular disease because of its anti-angiogenic properties systemically and ocular application (25). sEphB4-HSA was created by fusing the C-terminus of sEphB4 with albumin to improve its halflife. Albumin fusion protein has favorable pharmacokinetics in systemic circulation such that less frequent administration is feasible with equal or greater efficacy (30). We also determined that both EphrinB2 and EphB4 are expressed in choroidal vessels of adult rats (19), suggesting that Eph RTK and its ligand may have a role in vascular remodeling at disease sites in adult life in addition to their role in embryonic angiogenesis. In the current study, we showed that intravitreal injection of the sEPhB4 inhibited pathological angiogenesis in murine laser-induced $\mathrm{CNV}$ model. Our study demonstrated the effect of the sEphB4 on rats $\mathrm{CNV}$ using three methods: FA, lesion volume and lesion histology. Our results showed significant inhibitory effects on FA leakage (62.5\% reduction), CNV volume (57\% reduction) compared to PBS injections (31). Histologic analysis showed that the
$\mathrm{CNV}$ lesions were significantly reduced in the rats with the sEphB4 injection compared with PBS application. Our result is the similar to the inhibition of angiogenesis by the sEphB4 in other system such as mouse tumor models and early human trials (30).

Besides the stimulation in the formation of new vessels by angiogenic growth factors and cytokines (32), the interaction between vascular endothelial cells and extracellular matrix is required for vessel integrity (33). In particular FN localizes to the vascular wall for vascular stability. Importantly, knocking down the gene of FN inhibits VEGF expression and endothelial cells proliferation (34). Our results show that $\mathrm{FN}$ expression was abundant in the $\mathrm{CNV}$ lesion with PBS application, however, the immunoreactivity of FN was reduced by the injection of sEphB4; in addition, the double labeling (Figure 1E,F) showed that binding of FN to EphB4 is reduced after EphB4 administration in the lesion of rats $\mathrm{CNV}$. Taken together, it is plausible that the lack of FN binding to EphB4 due to competitive inhibition by sEphB4 is an additional possible mechanism that interferes with EphB4-EphrinB2 interactions, inhibiting VEGF expression 

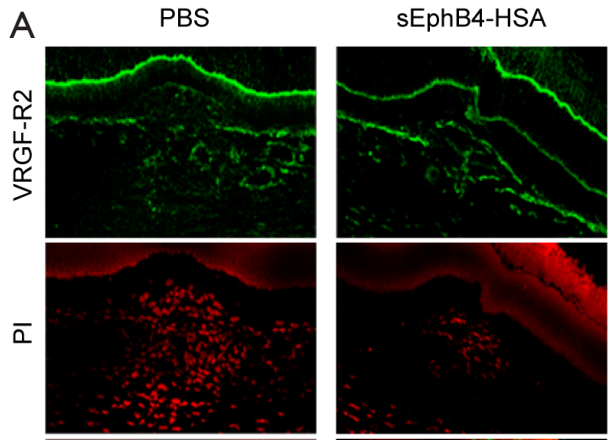

B
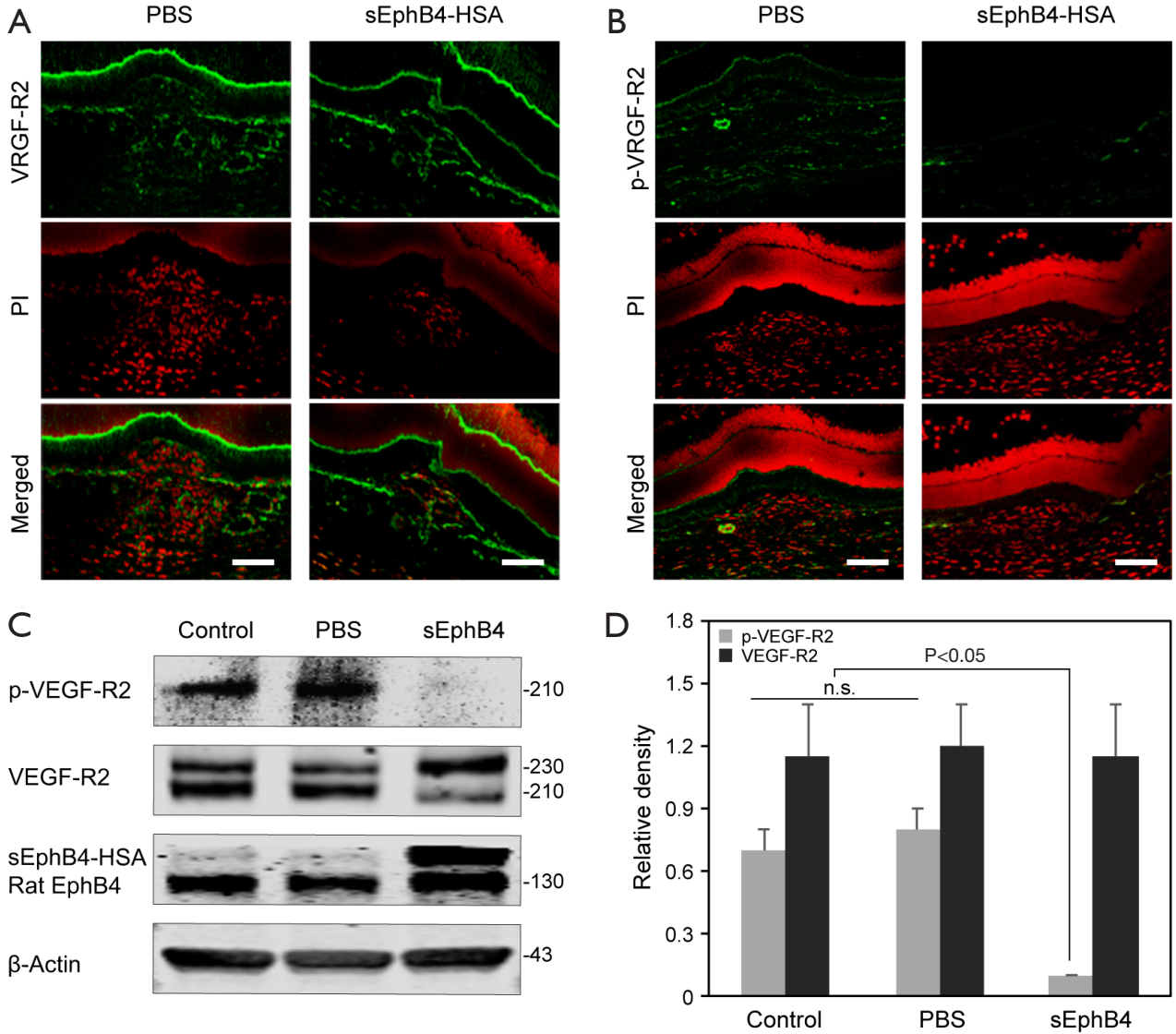

Figure 4 Treatment of sEphB4 inhibits VEGFR2 (vascular endothelial growth factor receptor 2) phosphorylation in rat retina. The expression of VEGFR2 (A) and pVEGFR2 (phosphorylated vascular endothelial growth factor receptor 2) (B) in laser induced CNV lesion after laser days 14. VEGFR2 stained as green and nuclear counterstain as red. There is no significant difference in the expression of total VEGFR2 between PBS (phosphate-buffered saline) and sEphB4-HSA injection (A); however, the pVEGFR2 expression was much weaker (B) in sEphB4-HSA injected rats compared with controls. Scale bar: $25 \mu \mathrm{m}$. (C) Western blot analysis showed phosphorylation of VEGFR2 in the eye with the sEphB4 injection was significantly reduced compared with PBS group, however, total VEGFR2 protein levels remained the same. (D) Quantitative analyses of the data were representative of three independent experiments. Values represent mean \pm SD, and the $\mathrm{P}$ value was shown in the figure.

which ultimately impairs CNV.

VEGF is the key driver in angiogenesis, through activation of VEGFR2 expressed exclusively on vascular endothelial cells. VEGF promotes endothelial cell proliferation, migration and thus new vessels formation (35). Inhibition of VEGFR2 signaling results in suppression of experimental $\mathrm{CNV}$ in animal models (36). In the current study, we found pVEGFR2 expression was markedly reduced after sEphB4-HSA treatment in CNV lesions measured by both in situ staining and western blotting with no apparent change in total VEGFR2 levels. These data indicate that the sEphB4 inhibits VEGFR2 phosphorylation consistent with the known role of EphrinB2 in facilitating VEGF mediated phosphorylation of VEGFR2. Furthermore, EphrinB2 co-localizes with VEGFR2 in endothelial cells and lack of EphrinB2 attenuates VEGF signaling. sEphB4 binds EphrinB2 and appears to diminish VEGFR2 activation. The sEphB4 thus in part acts through VEGF signaling in suppressing rat CNV. EphB4 also binds to FN. sEphB4-HSA localizes to FN in the vessel wall for increased delivery to the site of action and also prolonged retention time in the eye. Our results suggest that the sEphB4 may be a therapeutically potential for the treatment of angiogenesis related eye diseases. Our study for the 
inhibition of CNV by sEphB4 was limited to 30 rats in one specific strain, much more work has to be done, for example the dynamic of sEphb4 in vitreous and retina, and the safety of the reagent to retina and other intraocular tissues in term of ultrastructure analysis and electroretinography (ERG), how is the response of sEphB4 injection to other animals such as rabbit and monkey $\mathrm{CNV}$.

\section{Acknowledgments}

Funding: None.

\section{Footnote}

Reporting Checklist: The authors have completed the ARRIVE reporting checklist. Available at http://dx.doi. org/10.21037/atm-20-3810

Data Sharing Statement: Available at http://dx.doi. org/10.21037/atm-20-3810

Conflicts of Interest: All authors have completed the ICMJE uniform disclosure form (available at http://dx.doi. org/10.21037/atm-20-3810). The authors have no conflicts of interest to declare.

Ethical Statement: The authors are accountable for all aspects of the work in ensuring that questions related to the accuracy or integrity of any part of the work are appropriately investigated and resolved. All procedures of the animal experiment were conducted in accordance with the ARVO Statement for the Use of Animals in Ophthalmic and Vision Research and approved by the University of Southern California Animal Use Committee.

Open Access Statement: This is an Open Access article distributed in accordance with the Creative Commons Attribution-NonCommercial-NoDerivs 4.0 International License (CC BY-NC-ND 4.0), which permits the noncommercial replication and distribution of the article with the strict proviso that no changes or edits are made and the original work is properly cited (including links to both the formal publication through the relevant DOI and the license). See: https://creativecommons.org/licenses/by-nc-nd/4.0/.

\section{References}

1. Grossniklaus HE, Green WR. Choroidal neovascularization. Am J Ophthalmol 2004;137:496-503.

2. Shaw PX, Stiles T, Douglas C, et al. Oxidative stress, innate immunity, and age-related macular degeneration. AIMS Mol Sci 2016;3:196-221.

3. Ozaki E, Campbell M, Kiang AS, et al. Inflammation in age-related macular degeneration. Adv Exp Med Biol 2014;801:229-35.

4. de Oliveira Dias JR, Rodrigues EB, Maia M, et al. Cytokines in neovascular age-related macular degeneration: fundamentals of targeted combination therapy. Br J Ophthalmol 2011;95:1631-7.

5. Chan N, He S, Spee CK, et al. Attenuation of choroidal neovascularization by histone deacetylase inhibitor. PLoS One 2015;10:e0120587.

6. Grisanti S, Zhu Q, Tatar O, et al. Differential expression of vascular endothelial growth factor-a isoforms in neovascular age-related macular degeneration. Retina 2015;35:764-72.

7. Kwak N, Okamoto N, Wood JM, et al. VEGF is major stimulator in model of choroidal neovascularization. Invest Ophthalmol Vis Sci 2000;41:3158-64.

8. Turgut B, Tanyildizi R. The frequency of the nonresponsiveness to intravitreal injection of the antivascular endothelial growth factor agent in neovascular age related macular degeneration. In J Ophthalmic Pathol 2013;2:1-4

9. Nalluri SR, Chu D, Keresztes R, et al. Risk of venous thromboembolism with the angiogenesis inhibitor bevacizumab in cancer patients: a meta-analysis. JAMA 2008;300:2277-85.

10. Arevalo JF, Maia M, Flynn HW Jr, et al. Tractional retinal detachment following intravitreal bevacizumab (Avastin) in patients with severe proliferative diabetic retinopathy. $\mathrm{Br} \mathrm{J}$ Ophthalmol 2008;92:213-6.

11. Day S, Acquah K, Mruthyunjaya P, et al. Ocular complications after anti-vascular endothelial growth factor therapy in medicare patients with age-related macular degeneration. Am J Ophthalmol 2011;152:266-72.

12. Salvucci O, Tosato G. Essential roles of EphB receptors and EphrinB ligands in endothelial cell function and angiogenesis. Adv Cancer Res 2012;114:21-57.

13. Chen $\mathrm{Y}$, Zhang $\mathrm{H}$, Zhang Y. Targeting receptor tyrosine kinase EphB4 in cancer therapy. Semin Cancer Biol 2019;56:37-46.

14. Wilkinson DG. Multiple roles of EPH receptors and ephrins in neural development. Nat Rev Neurosci 2001;2:155-64.

15. Gale NW, Baluk P, Pan L, et al. Ephrin-B2 selectively 
marks arterial vessels and neovascularization sites in the adult, with expression in both endothelial and smoothmuscle cells. Dev Biol 2001;230:151-60.

16. Pasquale EB. Eph receptor signaling casts a wide net on cell behaviour. Nat Rev Mol Cell Biol 2005;6:462-75.

17. Swift MR, Weinstein BM. Arterial-venous specification during development. Circ Res 2009;104:576-88.

18. Uhl C, Markel M, Broggini T. EphB4 mediates resistance to antiangiogenic therapy in experimental glioma. Angiogenesis 2018;21:873-81.

19. He S, Ding Y, Zhou J, et al. Soluble EphB4 regulates choroidal endothelial cell function and inhibits laserinduced choroidal neovascularization. Invest Ophthalmol Vis Sci 2005;46:4772-9.

20. Umeda N, Ozaki H, Hayashi H, et al. Expression of ephrinB2 and its receptors on fibroproliferative membranes in ocular angiogenic diseases. Am J Ophthalmol 2004;138:270-9.

21. He S, Kumar SR, Zhou P, et al. Soluble EphB4 inhibition of PDGF-induced RPE migration in vitro. Invest Ophthalmol Vis Sci 2010;51:543-52.

22. Shi S, Liu J, Joshi SB, et al. Biophysical characterization and stabilization of the recombinant albumin fusion protein sEphB4-HSA. J Pharm Sci 2012;101:1969-84.

23. Tanemura M, Miyamoto N, Mandai M, et al. The role of estrogen and estrogen receptor $\beta$ in choroidal neovascularization. Mol Vis 2004;10:923-32.

24. Du E, Li X, He S, et al. The critical role of the interplays of EphrinB2/EphB4 and VEGF in the induction of angiogenesis. Mol Biol Rep 2020;47:4681-90.

25. Brar M, Cheng L, Yuson R, et al. Ocular safety profile and intraocular pharmacokinetics of an antagonist of EphB4/ EphrinB2 signaling. Br J Ophthalmol 2010;94:1668-73.

26. Courtoy PJ, Boyles J. Fibronectin in the microvasculature: localization in the pericyte-endothelial interstitium. J

Cite this article as: $\mathrm{He} \mathrm{S}$, Ouyang S, Li X, Ma B. Inhibition of laser induced rats choroidal neovascularization by intravitreous injection of sEphB4-HSA. Ann Transl Med 2021;9(1):18. doi: 10.21037/atm-20-3810
Ultrastruct Res 1983;83:258-73.

27. Bergers G, Song S. The role of pericytes in blood-vessel formation and maintenance. Neuro Oncol 2005;7:452-64.

28. Liu Z, Fan F, Wang A, et al. Dll4-Notch signaling in regulation of tumor angiogenesis. J Cancer Res Clin Oncol 2014;140:525-36.

29. Abéngozar MA, de Frutos S, Ferreiro S, et al. Blocking ephrinB2 with highly specific antibodies inhibits angiogenesis, lymphangiogenesis, and tumor growth. Blood 2012;119:4565-76.

30. Li X, Choi WW, Yan R, et al. The Differential Expression of EphB2 and EphB4 Receptor Kinases in Normal Bladder and in Transitional Cell Carcinoma of the Bladder. PLoS One 2014;9:e105326.

31. Hollanders K, Van Bergen T, Van de Velde S, et al. Bevacizumab revisited: its use in different mouse models of ocular pathologies. Curr Eye Res 2015;40:611-21.

32. Barrientos S, Stojadinovic O, Golinko MS, et al. Growth factors and cytokines in wound healing. Wound Repair Regen 2008;16:585-601.

33. Davis GE, Senger DR. Endothelial Extracellular Matrix: Biosynthesis, Remodeling, and Functions During Vascular Morphogenesis and Neovessel Stabilization. Circ Res 2005;97:1093-107.

34. Hielscher A, Ellis K, Qiu C, et al. Fibronectin Deposition Participates in Extracellular Matrix Assembly and Vascular Morphogenesis. PLoS One 2016;11:e0147600.

35. Basu S, Nagy JA, Pal S, et al. The neurotransmitter dopamine inhibits angiogenesis induced by vascular permeability factor/vascular endothelial growth factor. Nat Med 2001;7:569-74.

36. Gu L, Chen H, Tuo J, et al. Inhibition of experimental choroidal neovascularization in mice by anti-VEGFA/ VEGFR2 or non-specific siRNA. Exp Eye Res 2010;91:433-9. 